\title{
Magnetospheric Convection
}

\author{
G. ATKINSON \\ Herzberg Institute of Astrophysics, National Research Council of Canada, \\ 100 Sussex Drive, Ottawa, Ontario KIA OR6, Canada
}

(Received August 6, 1990; Revised December 4, 1990)

\begin{abstract}
Ideally, in the convection problem we should start at the bow shock and solve self-consistently for energy, momentum and plasma transfer in the complete downstream system. The state of the art is to study individual regions (magnetosheath, tail lobes, high-beta plasma sheet, and dipolar region) and make assumptions about the boundary conditions connecting them to neighbouring regions. Plasma sheath convection can be approximated by the model of a blunt body immersed in a supersonic or super-Alfvénic flow with some degree of success. Improvements need to include the effects of the daysidemerging and the nightside expansion fan sections of the magnetopause on flow in the plasma sheath. Convection in the dipolar region is fairly well understood in terms of the calculation scheme proposed by VASYLIUNAS (1972), but a self-consistent determination of the latitude of the high-latitude boundary is needed as well as a better understanding of boundary conditions. Understanding of convection in the high-beta plasma sheet and lobe is still very poor. The energy source is electromagnetic and can be represented as Poynting vector flow from the highlatitude magnetopause. We are still very uncertain as to the energy sink and the plasma sources and sinks in the plasma sheet. In the tail lobes, selfconsistency is needed between the field-line topology (including twists and open-closed configuration) and the location of and merging rate at $\mathrm{X}$ lines. Non-MHD processes are important at the boundaries between all the above regions.
\end{abstract}

\section{The System and the Ideal Solution}

A measure of our understanding of a physical system, such as the magnetosphere, is the extent to which we can predict its behaviour starting from basic physics (in this case, plasma physics) and a set of initial and boundary conditions. In this paper, I shall attempt to evaluate how well we understand the magnetosphere.

Figure 1 shows the system of interest. For the purposes of this paper, it is divided into the five regions indicated in the bottom half of the figure. These are the solar wind, the magnetosheath, and then within the magnetosphere: the tail lobes, the high-beta plasma sheet and the dipolar region. The high-beta plasma sheet consists of closed magnetic flux tubes which are substantially distorted from a dipole-like form. Boundaries between these regions include: (i) the bow shock which, because it is a fast shock, is thin and clearly defined. (ii) the magnetopause which, in its simplest form, consists of a dayside merging configuration (X-line, slow shocks, magnetic islands?) connected to a downstream slow expansion fan which is not a sharp discontinuity, (iii) the plasma sheet boundary layer, also not a sharp discontinuity, which may be the result of merging at the nightside $\mathrm{X}$ line shown in the figure and (iv) the boundary between significantly distorted field lines and almost-dipolar field lines. The two X-lines shown in the figure, one on the 


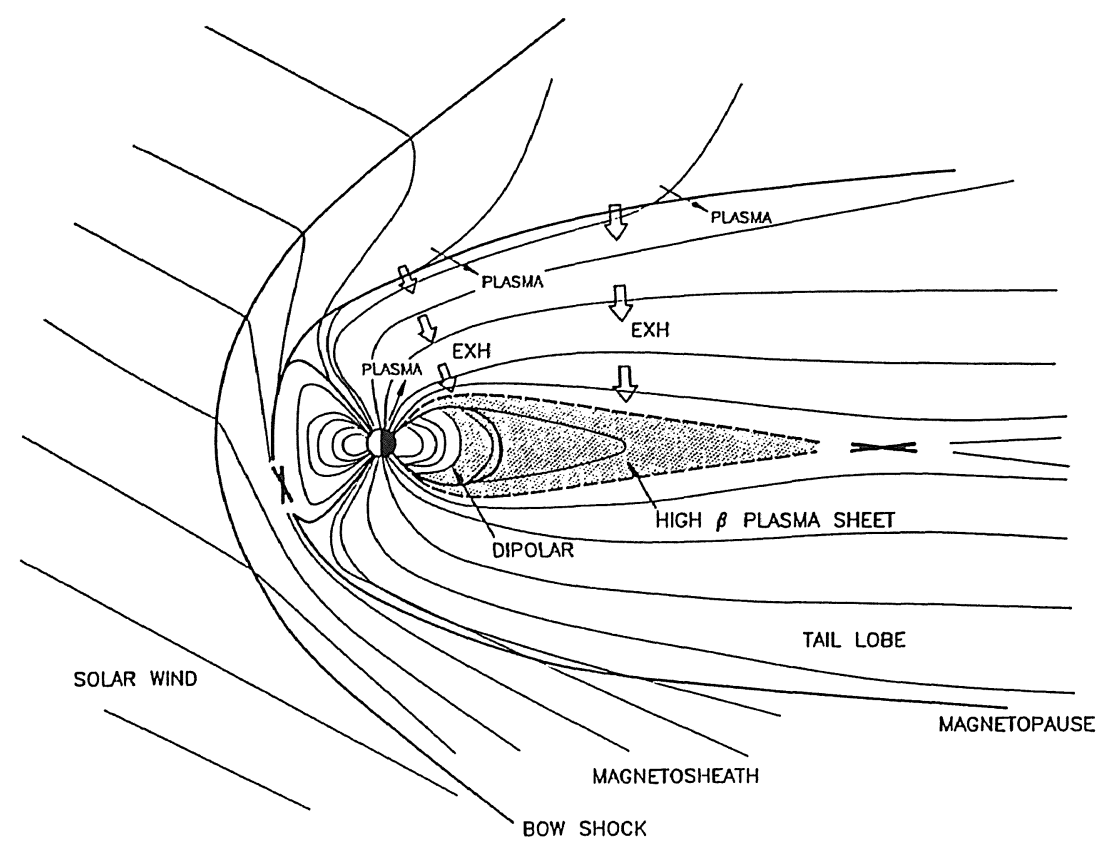

Fig. I. Noon-midnight section showing the four regions: magnetosheath, tail lobe, high beta plasma sheet and dipolar, and the two $\mathrm{X}$ lines (the minimum possible). In the upper half, Poynting vectors and plasma flow are shown.

dayside and one on the nightside, are the minimum required for convection to occur according to the generally accepted picture. There may in fact be multiple $\mathrm{X}$ lines and magnetic islands on both the dayside and the nightside.

The "convection problem" originates in the pioneer papers by AXFORD and HINES (1961) and Dungey $(1961,1963)$. It addresses the flow of mass and energy, and the transfer of momentum throughout the complete system. Ideally the solution should be a self-consistent one which includes the complete system downstream of the bow shock. This is because all the other boundaries involve energy and mass flow, and momentum transfer both ways. Only the solar wind is not significantly affected by the downstream region due to its super-alfvénic nature. (Upstream whistler wave and fast particle effects are small in terms of momentum transfer.) It should be noted that this problem is complicated by the fact that it is a free-boundary problem. Processes in the magnetosphere that produce currents change the shape of the magnetopause which in turn changes the shape of the shock. Thus the energy and mass extraction from the solar wind is coupled to processes inside the magnetosphere.

A solution of the complete system is beyond the state of the art. Thus we look at the four regions (magnetosheath, lobe, high-beta plasma sheet, dipolar) individually, make assumptions about the boundary locations and boundary conditions provided by the neghbouring regions, and calculate the convection inside each region. We shall now look at the state of the art in each of these regions in turn. 


\section{The Magnetosheath}

Calculations of shock position and flow and other plasma and field parameters in the magnetosheath generally assume supersonic gasdynamic or super-Alfvénic magnetohydrodynamic flow around a blunt body, the magnetosphere (e.g. SPREITER et al., 1965; DRYer and HECKMAN, 1967; SPREITER and AlKSNe, 1969; SLAVIN et al., 1983). The hydrodynamic calculations are well developed and give predictions of flow, density and temperature throughout the magnetosheath. The calculations have produced shock locations and shape in good agreement with observations. The extent of Agreement with the other predictions has not been as well studied, but it is clear that magnetic field and plasma effects become important, particularly in the subsonic (sub-Alfvenic) region. The ability of the hydrodynamic theory to predict the observations is consistent with the dominant process being the compression of plasma and field on the dayside and its subsequent expansion downstream around the magnetosphere. Less than or of the order of, ten percent of the energy incident upon a $15 \mathrm{R}_{\mathrm{E}}$ circle is dissipated in the near-earth magnetosphere on the average.

Improvements to the above approach are difficult and may be possible only through a series of simulations of increasing accuracy. Improvements require inclusion of the coupling between the magnetosheath and the magnetosphere. This coupling is quite complicated. In the slow-mode expansion region of the magnetopause (adjacent to the magnetotail), energy and mass flow are from the magnetosheath to the magnetotail, and momentum is transferred both ways. In the merging configuration region of the magnetopause (the dayside), theories indicate that a boundary layer is produced with energy and mass inflow from both the magnetosheath and magnetosphere and momentum is interchanged with each. The transition from the dayside merging configuration to the downstream slow expansion wave is not well understood.

\section{The Magnetosphere-General Considerations}

Before examining the individual regions we shall take a brief look at energy, mass and momentum transfer in the whole magnetosphere.

The behaviour of electromagnetic energy can be described in terms of the divergence of the Poynting vector:

$$
\nabla \cdot\left(\frac{\boldsymbol{E} \times \boldsymbol{B}}{\mu_{0}}\right)=\frac{\partial}{\partial t}\left(\frac{B^{2}}{2 \mu_{0}}\right)+\boldsymbol{J} \cdot \boldsymbol{E} .
$$

All terms are major contributors in the magnetosphere. The first term on the RHS represents the storage and release of magnetic energy. It is of major importance in the tail lobes and plasma sheet where the storage and release of magnetic energy is a major contributor to magnetospheric substorms. The last term on the RHS of Eq. (1) represents transfer of electromagnetic energy to the plasmas. It is of major importance in describing energization of the plasma sheet and ring current plasma, and also in the ionosphere where Joule heating occurs. On the magnetopause it describes the "generator" as plasma kinetic energy is transformed into electromagnetic energy. In the plasma sheet and ring current, 
where the plasmas are hotter, transport of energy by plasma convection also becomes important.

The overall picture is that the energy flow is dominated by electromagnetic energy inflow from the magnetopause through the tail (as shown by the open arrows in the upper half of Fig. 1). In the tail, there is time dependent storage and release of energy associated with susbtorms. The poynting vector flow continues into the plasma sheet and dipolar region, where plasma energization occurs. Subsequent energy inflow occurs into the nightside and around the flanks to the dayside magnetopause, both as electromagnetic and plasma transported energy. There is dissipation in the plasma and ionosphere. However, as will be discussed in the section on the high-beta plasma sheet, this is not a complete picture since other major energy sinks must also exist for the energy entering the plasma sheet.

The mass (plasma) flow in the magnetosphere is not well understood. Three possible sources are generally discussed in the literature. The first is flow as part of the highlatitude boundary layer through the magnetopause. The suggested subsequent flow is then somewhat similar to the energy flow except that initially there is a large antisunward component in the tail (this is illustrated by the solid arrows in Fig. 1). A second possible source of plasma is the flanks of the plasma sheet. A third source is the ionosphere and there is considerable evidence for the existence of particles of ionospheric origin in the plasma sheet as well as observations of upward flowing ion beams and conics at low altitudes (again illustrated by solid arrows in Fig. 1). Sinks suggested for the plasma include the dayside magnetopause in the region dominated by the merging configuration, precipitation into the ionosphere, loss through the dawn and dusk flanks, and downtail escape.

Finally, it should be noted that the convection problem requires solution of the momentum equations (or equivalently the equations of current closure). This in turn requires a knowledge of magnetopause currents and hence of both shear and normal

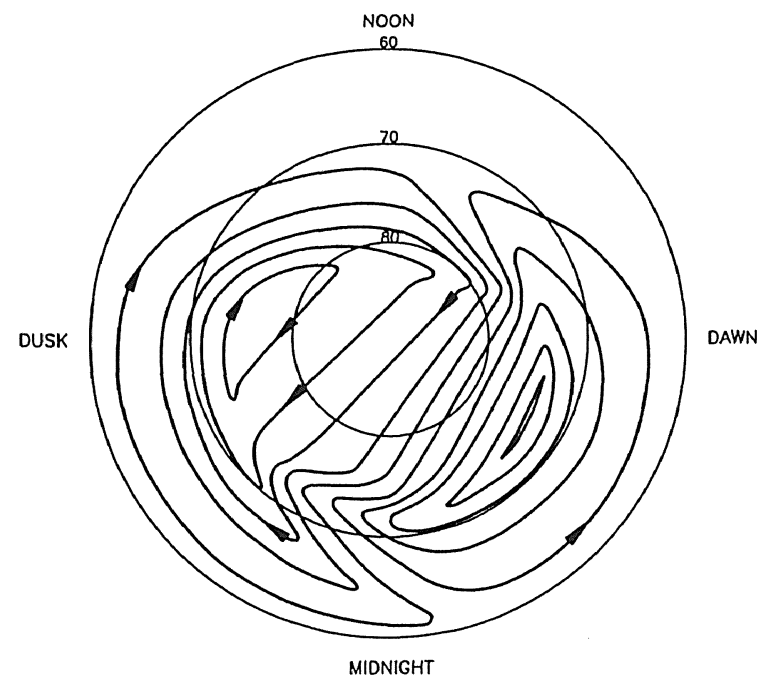

Fig. 2. Polar cap view showing a representative set of electric equipotentials. This set is for the northern (southern) hemisphere when $B_{y}>0\left(B_{y}<0\right)$ and $B_{z}<0$. Adapted from HePPNER and MAYNARD (1987). 
stresses at the magnetopause. Generally, normal stresses dominate and a full knowledge of these should allow a reasonably accurate solution of the problem. This can be seen in that the existence of convection and internal currents in the magnetosphere changes the topology (lowering or raising the cusps, changing the flaring angle and field strength) and hence produces the self-consistent shape which closes all internal currents and provides the correct energy inflow.

What are the observations that the theories aim to reproduce? A common way to present results is to show patterns of electric-field equipotentials. Figure 2 (from HEPPNER and MAYNARD, 1987) shows a typical pattern of the equipotentials mapped along magnetic field lines to the ionosphere. The pattern depends strongly on the orientation of the interplanetary magnetic field (IMF) and the set in Fig. 2 is valid for the northern (southern) hemisphere when $B_{y}>0\left(B_{y}<0\right)$ and $B_{z}<0$. The interested reader is referred to papers by HEPPNER (1977), BURKE et al. (1979), BURCH et al. (1985), REIFF and BURGH (1985), HEPPNER and MAYNARD (1987) and GUSSENHOVEN (1988) for more details of the dependence of the patterns on the IMF. For $B_{z}>0$, the patterns are quite complicated and there is controversy over the global-scale patterns corresponding to the observations which are taken in limited areas.

\section{The Dipolar Region}

We now examine each of the parts of the magnetosphere in more detail, starting with the dipolar region because it is the best understood.

Figure 3 shows a flow chart for a self-consistent calculation scheme for this region. From the hot particle distribution in a model magnetic field, the gradient and curvature drift and hence the perpendicular currents can be calculated. The divergence of the latter gives the Birkeland (field-aligned) currents. The requirement for closure of Birkeland currents in the ionosphere specifies the electric fields, and the electric fields and gradient and curvature drifts can be used to determine the evolution of the hot particle distribution. The basic physics was identified by FEJER (1964). This calculation scheme was proposed by VASYLIUNAS (1972) and is the basis of the Rice University computer model (e.g. HAREL and Wolf, 1976). Some simplifications and assumptions have led to the other solutions also (e.g. SOUTHWOOD, 1977; CROOKER and SISCOE, 1981; SENIOR and BLANK, 1984; ATKINSON, 1986). The calculated flows give reasonable agreement with observed auroral zone convection of the type illustrated in Fig. 2.

In its simplest form the model assumes a model for the magnetic field, equipotential

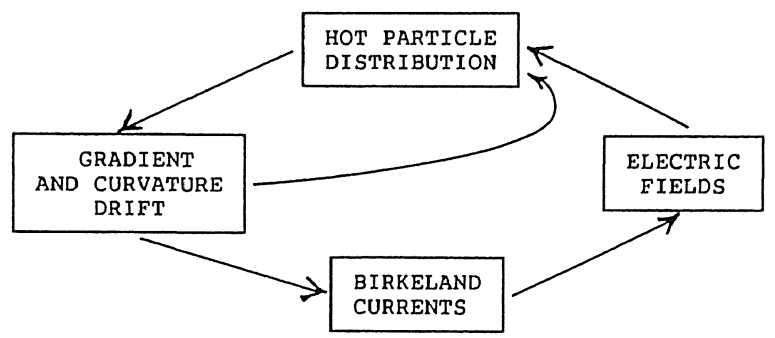

Fig. 3. A self-consistent calculation scheme for convection in the dipolar region. 
field lines, a model for ionospheric conductivity, a high-latitude boundary location, and boundary conditions. Additional complications studied have included field-aligned potential drops and determination of ionospheric conductivity from precipitation and solar zenith angle.

A number of areas still need some improvement. A self-consistent magnetic field should be included in the model calculations. In addition, the common assumption for high-latitude boundary conditions of equipotentials being anti-symmetric about midnight is doubtful (ATKINSON, 1986), since non-MHD processes are an essential part of inflow in the tail as will be discussed later. It can be seen in Fig. 2 that symmetric nightside boundary conditions are not consistent with many observations.

Even more important, in the opinion of the author, is the latitudinal location of the high-latitude boundary for this region. A thick Alfvén layer (corresponding to the sum of the thin Alfvén layers for each particle energy) is an essential part of the physics of the dipolar region. In the Alfvén layer, curvature and gradient drift of particles become as important as the electric field drift in moving particles and energy around and the layer determines the earthward edge of the plasma sheet. Birkeland currents are produced within the layer by the divergence of the curvature and gradient drifts. The selection of a latitude for the high-latitude boundary determines whether most of the flow to the dayside occurs within the Alfvén layer (and hence for uniform ionospheric conductivity obeys $\left.\nabla^{2} \phi=J_{\|}\right)$or poleward of it $\left(\nabla^{2} \phi=0\right)$. The two cases are subject to different physics. ATKINSON (1988) argued that the boundary should be at or near the poleward edge of the Alfvén layer.

\section{High Beta Plasma Sheet}

Our understanding of the high-beta plasma sheet is very limited and we are still at the stage of trying to determine which processes are dominant.

The major energy input is believed to be the Poynting vector energy flow, illustrated by the open arrows in Fig. 1, from the magnetopause through the lobes to the plasma sheet. In the plasma sheet, energization of the plasma occurs corresponding to the $\boldsymbol{J} \cdot \boldsymbol{E}$ term of Eq. (1). As noted earlier, the existence of substorms indicates a strong timedependence as the electromagnetic energy is stored and then released in the tail.

It was pointed out by ERICSON and WOLF (1980) and SCHINDLER and BIRN (1982) that there is an energy problem. The above process creates too much energy in the plasma to allow convection of these flux tubes into the nightside dipolar region for an $\mathrm{X}$ line at distances of the order of $100 \mathrm{R}_{\mathrm{E}}$ down the tail if no energy losses occur. Presumably, a loss mechanism does occur and it is non-MHD in nature. suggested mechanisms include periodic plasmoid or magnetic island ejection down the tail and energetic particle losses through the flanks. Other known sinks for the plasma sheet energy include precipitation into the ionosphere and convection into the dipolar region. These last two are believed to be too small to provide a sink for all the energy entering the plasma sheet.

The origins of particles is also poorly understood. It should be noted that the sources of particles and energy are probably different. Suggested particle sources include solar wind entry through the high-latitude boundary layer, solar wind entry through the dawn and dusk flanks, and particles escaping from the ionosphere. There are many observations of ionosphere-origin particles both in the plasma sheet (see reviews by WILLIAMS, 1987, and HoRwITZ, 1987) and at lower altitudes where they can be seen streaming upwards as 
ion beams, conics and polar wind (YAU et al., 1985).

Further unresolved problems associated with the high-beta plasma sheet include the existence of the plasma-sheet boundary layer (WILLIAMS, 1981; EASTMAN et al., 1985), a region at the high-latitude edge of the plasma sheet where ions are observed to stream parallel and anti-parallel to magnetic field lines. In another paper in this conference, Hones suggests that the layer may be the result of reconnection at nightside $X$ lines of the type shown in Fig. 1. Other problems include the existence of magnetic islands or flux ropes embedded in the plasma sheet and tail. There are a number of reports of observations of these (e.g. NISHIDA et al., 1986) and also predictions resulting from theory and simulations (FU and LEE, 1986; LEE et al., 1987). A final as yet unsettled major question for the nightside plasma sheet (which is most likely related to the energy problem) is whether the convection is essentially time-dependent. What is the nature and cause of magnetospheric substorms? It seems likely that until this basic question is answered out understanding of convection in the plasma sheet will remain severely limited.

\section{The Tail Lobes}

A recent review by the author (ATKINSON, 1990) discusses the convection and topology of this region in more depth. The approach taken here is to make the tail-lobe section of this paper complementary to the above review, although some overlap is inevitable. The above name for this region is perhaps strictly applicable only for southward IMF, since there is a body of observations that indicates that some highlatitude field lines may be closed for other orientations of the IMF.

Convection in the polar cap is still poorly understood for the IMF not directly southward. Consider a pattern of electric equipotentials, such as shown in Fig. 2. The source location (flow into the polar cap) and the sink location (outflow) are determined by the mapping of $X$ lines in the outer magnetosphere, since it is at $X$ lines that field lines are opened or closed. However the location of $\mathrm{X}$ lines is dependent on the topology and vice versa. Thus a solution is required with a self-consistent description of $\mathrm{X}$ lines and topology.

The other major effect for polar cap convection arises from the fact that Birkeland currents (or the twisting of flux tubes) are a necessary and unavoidable part of the problem. For example a $y$ component of the IMF causes field lines to wrap around the polar-cap flux tube after they are opened by dayside merging. This creates the type of twist cartooned in Fig. 4 at the left. In this type of twisting. Birkeland currents are expected to be distributed within the flux tube, although, due to time constants involved (ATKINSON, 1990), they should be strongest on freshly merged flux tubes and decay as the time since merging increases. The cartoon on the right side of Fig. 4 illustrates that the polar cap flux may be split, under some circumstances, into more than one flux tube, and flux tubes can be twisted about each other to produce current sheets in the polar cap. One of the tubes may in fact be closed and time constants again are of importance in determining the decay of the twist and the Birkeland currents.

In summary, to solve for polar cap convection patterns, we need a self-consistent description of flux tube topology; including twists, and the locations of dayside and nightside $\mathrm{X}$ lines. Some interesting ideas on this problem have come from CROOKER (1979) and REIFF and BURCH (1985). In these works it is assumed that, for different 


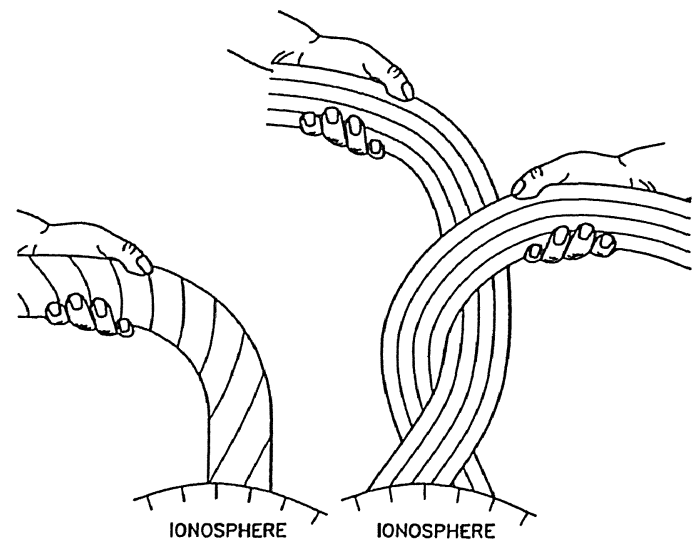

Fig. 4. Twists in polar flux tubes. Left: a twist about the flux tube axis corresponds to distributed birkeland currents. Right: two or more flux tubes twisted about each other corresponds to Birkeland current sheets.

orientations of the IMF in the $y z$ plane, merging occurs on the dayside: on closed field lines for the IMF near southward, on open field lines for the IMF near northward, and on both open and closed for other orientations of the IMF. The result is a hierarchy of convection cells which include:

1) Viscous cells (expected at the centres of the two cells shown in Fig. 2) driven by viscous-like interactions at the low latitude boundary layer and occurring entirely on closed field lines.

2) Merging cells, in which dayside merging opens field lines and nightside merging closes them, with a form similar to those in Fig. 2.

3) Lobe cells, in which merging on open field lines creates a twist in the polar cap flux tube and the convection cell closes completely on open field lines.

4) Reclosure cells which involve (as do merging cells) dayside opening of closed tubes and nightside closing of open tubes, but with the difference that the twist causes sunward convection of closed flux tubes across the centre of the polar cap to the dayside, and antisunward convection of open tubes at lower latitudes.

The above contains interesting models, but is not the only possibility. The number of cells existing at any time in the above models is as high as four. Some observers believe that all the observations can equally well be accounted for by only two cells which are distorted from the standard semi-circle shape (HEPPNER and MAYNARD, 1987). Such distortions might very well be expected due to twists in flux tubes.

\section{Comments}

In closing, the author wishes to make two comments. Non-MHD processes appear to be important at all the boundaries between regions and these need to be understood to improve our solutions. The non-MHD processes include the bow shock, merging at the magnetopause, merging or other processes producing the plasma-sheet boundary layer at the lobe-plasma sheet boundary, and merging or other non-MHD energy loss process at the inner edge of (and perhaps also within) the high-beta plasma sheet. 
The final comment concerns the discovery by ATKINSON et al. (1989) that quite complicated discrete auroral-arc dynamics can be explained by straight-forward $\mathrm{X}$ line/magnetic island kinematics. The proposed mapping between arcs and $X$ lines can be used to provide information, from arc locations, on X-line locations and magnetic island behaviour in the outer magnetosphere.

\section{REFERENCES}

ATKINSON, G., A theoretical model of convection with distributed Birkeland currents, J. Geophys. Res., 91, 5831-5838, 1986.

ATKINSON, G., The location of the high-latitude boundary for magnetospheric convection is not arbitrary, $J$. Geophys. Res., 93, 11,533-11,535, 1988.

AtKinson, G., Solar-terrestrial coupling poleward of the auroral zone, J. Geomag. Geoelectr., 42, 665-681, 1990.

Atkinson, G., F. Creutzberg, R. L. Gattinger, and J. S. Murphree, Interpretation of complicated discrete arc structure and behaviour in terms of X lines, J. Geophys. Res., 94, 5292-5302, 1989.

AXFORD, W. I. and C. O. HINES, A unifying theory of high latitude geophysical phenomena and geomagnetic storms, Can. J. Phys., 39, 1433-1464, 1961.

Burch, J. L., P. H. Reiff, J. D. Menietti, R. A. Heelis, W. B. Hanson, S. D. Shawhan, E. G. Shelliey, M. SugIURA, D. R. WeIMER, and. J. D. WINNINGHAM, IMF $B_{y}$-dependent plasma flow and Birkeland currents in the dayside magnetosphere 1. dynamics explorer observations, J. Geophys. Res., 90, 1577 1593, 1985.

Burke, W. J., M. C. Kelley, R. C. SAgAlyn, M. Smiddy, and S. T. LAi, Polar cap electric field structures with a northward interplanetary magnetic field, Geophys. Res. Lett., 6, 21-24, 1979.

Crooker, N. U., Dayside merging and cusp geometry, J. Geophys. Res., 84, 951-959, 1979.

CROOKER, N. U. and G. L. SisCOE, Birkeland currents as the cause of the low-latitude asymmetric disturbance field, J. Geophys. Res., 86, 11,201-11,210, 1981.

DRYER, M. and G. R. HECKMAN, On the hypersonic analogue as applied to planetary interaction with the solar plasma, Planet. Space Sci., 15, 515-546, 1967.

Dungey, J. W. Interplanetary magnetic field and auroral zones, Phys. Rev. Lett., 6, 47 48, 1961.

DungeY, J. W., Structure of the exosphere or adventures in velocity space, in Geophysics, The Earth's Environment, edited by C. DeWitt, J. Hieblot, and A. Lebeau, Gordon and Breach, New York, 1963.

EASTMAn, T. E., L. A. Frank, and C. Y. HUANG, The boundary layers as the primary transport regions of the earth's magnetotail, J. Geophys. Res., 90, 9541-9560, 1985.

ERICSON, G. M. and R. A. Wolf, Is steady convection possible in the earth's magnetotail?, Geophys. Res. Letl., 1, 897-900, 1980.

FEJER, J. A., Theory of the geomagnetic daily disturbance variations, J. Geophys. Res., 69, 123 135, 1964.

Fu, Z. F. and L. C. LEE, Multiple X line reconnection 2. The dynamics, J. Geophys. Res., 91, 13,373. 13,383, 1986.

GuSSENHOVEN, M. S., Low-altitude convection, precipitation and current patterns in the baseline magnetosphere, Rev. Geophys., 26, 792-808, 1988.

HAREl, M. and R. A. Wolf, Convection, in Physics of Solar-Planetary Environments: Proceedings of the International Symposium on Solar-Terrestrial Physics, Vol. 2, edited by D. J. WIlliams, ACj, Washington, D.C., 1976.

Heppner, J. P., Empirical model of high-latitude electric fields, J. Geophys. Res., 82, $11151125,1977$.

Heppner, J. P. and N. C. Maynard, Empirical high-latitude electric field models, J. Geophys. Res., 92, 4467-4489, 1987.

HorwITZ, J. L., Core plasma in the magnetosphere, Rev. Geophys., 25, 579-587, 1987.

LEE, L. C., Z. F. Fu, and S.-l. Akasofu, Multiple X-line reconnection in the earth's magnetosphere, in Magnetotail Physics, edited by A. T. Y. Lui, pp. 191-197, John Hopkins University Press, Baltimore, Maryland, 1987.

Nishida, A., M. Scholer, T. Terasawa, S. J. Bame, G. Gloekler, E. J. Smith, and R. D. Zwicki, Quasi stagnant plasmoid in the middle tail: a new pre-expansion phase phenomenon, J. Geophys. Res., 91, 4245-4255, 1986.

REIFF, P. H. and J. L. BURCH, IMF $B_{y}$-dependent flow and Birkeland currents in the dayside magnetosphere 2. a global model for northward and southward IMF, J. Geophys. Res., 90, 1595-1609, 1985. 
SCHINDLER, K. and J. BIRN, Self-consistent theory of time-dependent convection in the earth's magnetotail, $J$. Geophys. Res., 87, 2263-2275, 1982.

SENIOR, C. and M. BLANC, On the control of magnetospheric convection by the spatial distribution of ionospheric conductivities, J. Geophys. Res., 89, 261-284, 1984.

Slavin, J. A., R. E. Holzer, J. R. Spreiter, S. S. Stahard, and D. S. Chaussee, Solar wind flow about the terrestrial planets 2. Comparison with gas dynamic theory and implications for solar-planetary interactions, J. Geophys. Res., 88, 19-35, 1983.

SouthwOOD, D. J., The role of hot plasma in magnetospheric convection, J. Geophys. Res., 82, 5512-5520, 1977.

Spreiter, J. R. and A. Y. Alksne, Plasma flow around the magnetosphere, Rev. Geophys. Space Phys., 7, 11, 1969.

Spreiter, J. R., A. L. Summers, and A. Y. Alksne, Hydromagnetic flow around the magnetosphere, Planet. Space Sci., 14, 223, 1965.

VAsyliunAs, V. M., The interrelationship of magnetospheric processes, in Earth's Magnetospheric Processes, edited by B. M. McCormac, pp. 29-38, D. Reidel, Hingham, Massachusetts, 1972.

Williams, D. J., Energetic ion beams at the edge of the plasma sheet: ISEE I observations plus a simple explanatory model, J. Geophys. Res., 86, 5507-5518, 1981.

Williams, D. J., Ring current and radiation belts, Rev. Geophys., 25, 570-578, 1987.

Yau, A. W., E. G. Shelley, W. K. Peterson, and L. Lenchyshyn, Energetic auroral and polar ion outflow at DEl altitudes: magnitude, composition, magnetic activity dependence and long-term variations, $J$. Geophys. Res., 90, 8417-8432, 1985. 